\title{
Delivering Octadecylphosphonic Acid Self-Assembled Monolayers on a Si Wafer and Other Oxide Surfaces
}

\author{
Heng-Yong Nie,* Mary J. Walzak, and N. Stewart McIntyre \\ Surface Science Western, Room G-1, WSC, The University of Western Ontario, London, \\ Ontario N6A 5B7, Canada \\ Received: May 8, 2006; In Final Form: July 28, 2006
}

\begin{abstract}
We describe a simple experimental approach for delivering self-assembled monolayers (SAMs) of octadecylphosphonic acid (OPA) on many oxide surfaces using a nonpolar medium with a dielectric constant around 4 (e.g., trichloroethylene). This approach readily results in the formation of full-coverage OPA SAMs on a wide variety of oxide surfaces including cleaved mica, Si wafer, quartz, and aluminum. Especially, the availability of delivering full-coverage OPA SAM on a Si wafer is unique, as no OPA SAMs at all could be formed on a Si wafer when using a polar OPA solution. The reason a nonpolar solvent is superior lies in the very fact that the hydrophilic OPA headgroup tends to escape from the nonpolar solution and is thus enriched at the medium-air interface. It is these OPA headgroups seeking a hydrophilic surface that make possible the well-controlled OPA monolayer on an oxide surface.
\end{abstract}

\section{Introduction}

Amphiphilic organic molecules dissolved in a solvent can be adsorbed onto a solid substrate immersed in a solution, resulting in the formation of an ordered and oriented assembly of the molecules. ${ }^{1-8}$ This self-assembly of organic molecules should provide a simple path to fabricate ordered molecular structures. ${ }^{9}$ Self-assembled monolayers (SAMs) have aroused enormous interest in interdisciplinary research areas because they have many possible applications in the engineering of surfaces. ${ }^{10-13}$ There are two SAM systems that have been most studied: (1) SAMs formed from alkanethiols on gold surfaces through the specific and strong interaction between the sulfur atom and the gold atom ${ }^{1,4-8}$ and (2) SAMs formed from methylenetrichlorosilanes on oxide surfaces where the molecules have the ability to polymerize. ${ }^{2,3}$ The mechanisms for SAM formation, especially for the chemistry of alkanethiols on gold, ${ }^{8}$ have been thoroughly investigated in the last two decades. ${ }^{10-13}$ Molecular headgroups are immediately adsorbed on the substrate immersed in the polar solution, followed by a slower process of orientation of the molecular chains. Both nonpolar and polar solvents produced SAMs of similar quality for octadecyltrichlorosilane (OTS), ${ }^{2,3}$ and it appears that the polar solvent scheme has been adopted. Polymerization of siloxane on hydroxyl-terminated oxide surfaces seems to dominate the SAM formation mechanism, regardless of the solvent used. Formations of a full-coverage OTS monolayer either on a mica ${ }^{14}$ or on a single-crystal silicon ${ }^{15}$ substrate are believed to be a consequence of this particular ability of the OTS molecules to polymerize. ${ }^{16}$

Mica is frequently used as a demonstration substrate for octadecylphosphonic acid (OPA) SAM formation, for it is hydrophilic and atomically flat when freshly cleaved. In the past, studies show that polar solvents are appropriate vehicles for delivery of OPA monolayers on a mica substrate because a polar solvent dissolves OPA well and the solution wets the hydrophilic

* Author to whom correspondence should be addressed. E-mail: hnie@ uwo.ca. mica surface. ${ }^{16,17}$ OPA SAMs formed on it were found to be characterized by islandlike features or by a connected layer with pits ${ }^{16}$ or by something intermediate. ${ }^{17-19}$ However, full-coverage OPA SAMs on a mica surface have not been achieved in the polar solvent scheme, as judged by atomic force microscopy (AFM) imaging. ${ }^{16-19}$

Woodward et al. have pointed out the possibility of OPA serving as a general model system for investigating the fundamentals of SAM formation. ${ }^{16}$ This argument is supported by experimental findings on different interaction strengths between the OPA headgroup and different substrates. On one hand, organophosphonic acid dissolved either in a polar or in a nonpolar solvent has been known to have strong interactions with an oxidized $\mathrm{Al}$ surface.$^{20-23}$ For example, OPA monolayers have been deposited on an oxidized $\mathrm{Al}$ surface by immersing the substrate in either a polar ${ }^{20,21,23}$ or a nonpolar ${ }^{22}$ OPA solution. For phosphorus acids and short-chain organophosphonic acids, water as a delivery medium renders the acids chemically adsorbed on an oxidized $\mathrm{Al}$ surface. ${ }^{24}$ It is established that organophosphonic acid is bonded to the Al surface through $\mathrm{P}-\mathrm{O}-\mathrm{Al}^{21,24}$ via condensation reaction of the acidic hydroxyl groups with the Al hydroxyl groups, ${ }^{23,24}$ regardless of solvent used as the delivery medium.

On the other hand, it has been shown that no OPA SAMs at all could be formed on a $\mathrm{Si}$ wafer when a polar solvent was used, ${ }^{18}$ while under the same conditions, OPA SAMs are formed on the surface of an oxidized $\mathrm{Al}$ or a mica substrate. ${ }^{16-22}$ Hanson et al. have also pointed out that there was no OPA SAM formation by simply putting a Si substrate into an OPA solution in a polar solvent no matter how long was the immerse time (e.g., 3 days or 2 weeks), and they have shown that special steps including annealing the sample well above the melting point of OPA were needed to strongly bond OPA onto the $\mathrm{Si}$ substrate. ${ }^{25}$ It thus becomes clear that while the OPA headgroup reacts strongly with an oxidized $\mathrm{Al}$ surface, its interaction with an oxidized $\mathrm{Si}$ substrate appears so weak that immersing a $\mathrm{Si}$ substrate in a polar OPA solution alone is unable to form any 
OPA SAMs on a Si substrate, instead, liquidlike multilayers ${ }^{18}$ or aggregates ${ }^{25}$ form.

We describe in this work a method to specifically promote the interaction between the OPA headgroup and a hydrophilic substrate via use of appropriate nonpolar solvents. Our approach uses a spin-coating process with nonpolar solvents having a narrow range of dielectric constant near 4, such as trichloroethylene (TCE) and chloroform, to readily deliver full-coverage OPA SAMs on an oxidized Si wafer. We also show, in general, that such a nonpolar solvent is a more effective medium than a polar one for delivering coverage-controllable OPA SAMs on many hydrophilic oxide surfaces, such as oxidized $\mathrm{Al}$, sapphire, muscovite mica, and biotite. In case of Si substrate where this process has succeeded in producing a full-coverage OPA monolayer, the earlier approach using a polar solvent was unable to produce any monolayers. ${ }^{18}$ When an OPA monolayer was produced on a mica ${ }^{16,17,19}$ or a sapphire ${ }^{26}$ substrate using the polar solvent scheme, it was incomplete. We propose that the hydrophilic OPA headgroups in a nonpolar medium aggregate on the liquid surface making it possible for the spin-coating process to apply a "raft" of such aligned OPA headgroup; this provides a favorable environment for the hydrophilic headgroup to interact with the hydrophilic substrate, leading to a much faster growth rate for the formation of SAMs. Removal of excess solution from the surface is achieved, as a result of the spincoating process.

\section{Experimental Section}

Spin-Coating. Shown in Figure 1a is a schematic illustration for the method of spin-coating OPA monolayers on a hydrophilic surface via use of a nonpolar solvent (e.g., TCE) as the delivery medium. Throughout this article, we only show the OPA case; other organophosphonic acids are expected to work in the same manor as does OPA. When the nonpolar OPA medium is spread on a hydrophilic surface by the spin process, the hydrophilic OPA headgroups, concentrated and oriented at the surface of the liquid, are well positioned to interact with the surface. When the medium runs away from the hydrophilic surface, the hydrophilic OPA headgroups in the nonpolar solution continue to adhere to the hydrophilic substrate. Shown in Figure $1 \mathrm{~b}$ is the process whereby incomplete monolayers on such a surface become complete. If the medium is contacted with a preexisting hydrophobic OPA monolayer terminated by methyl groups, the hydrophilic OPA headgroups will not interact. Therefore, the action of delivering additional OPA solution to the surface leaves the existing monolayer intact and results in interaction only with the unoccupied part of the hydrophilic substrate. Through this additive mechanism, a full-coverage OPA SAM is achievable. Of course, a full-coverage monolayer may be formed by only one application of an OPA solution having a sufficiently high concentration onto the rotated substrate.

When inserting a hydrophilic substrate into a nonpolar OPA solution, an interface between the nonpolar medium and the substrate will be formed and the hydrophilic OPA headgroups will tend to concentrate at that newly created interface. Therefore, OPA SAM formation is also possible for dip-coating as well as immersion.

Materials and Sample Preparation. Crystalline powder of OPA $\left[\mathrm{CH}_{3}\left(\mathrm{CH}_{2}\right)_{17} \mathrm{P}(\mathrm{O})(\mathrm{OH})_{2}\right]$ was purchased from Alfa Aesar (93\% purity, Ward Hill, MA). The OPA powder was usually heated to $\sim 100{ }^{\circ} \mathrm{C}$ prior to use. This way, any influence caused by moisture to the OPA powder could be eliminated. This procedure was especially recommended in case that the OPA powder container had been previously opened for many times.
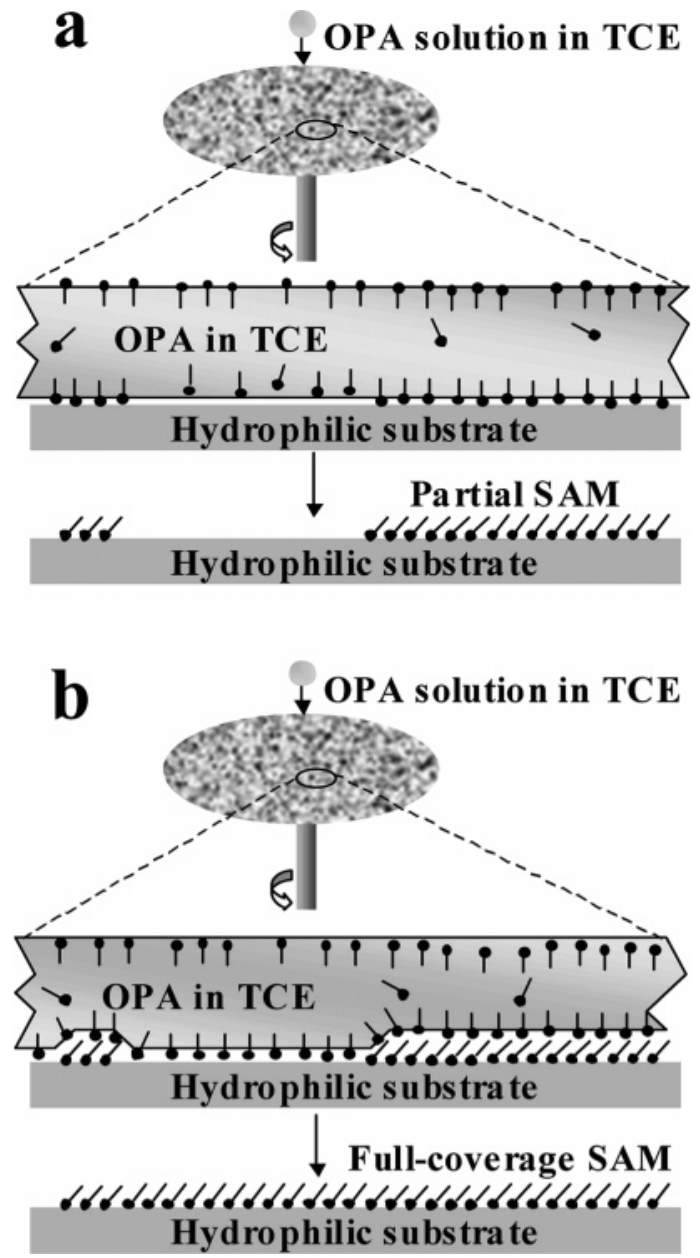

Figure 1. Schematic illustration of the concept of using an OPA solution in an appropriate solvent (e.g., TCE) to deliver (a) partial monolayers and (b) a full-coverage monolayer by applying increasing aliquots of the OPA solution to the spinning substrate. The hydrophilic OPA headgroup and the hydrocarbon tail are depicted as a filled circle and a line, respectively. The solvent is so chosen that the hydrophilic OPA headgroup aligns and concentrates on the solution surface. Note in (b) that the hydrophilic OPA headgroups over the existing monolayer will not interact with the terminating methyl groups.

A nominally $2 \mathrm{mM}$ OPA solution in TCE was used to prepare the OPA monolayers on various oxide surfaces. However, at room temperature and at this level of concentration, the OPA molecules were not completely dissolved in TCE and crystallinelike flakes were seen floating on the solution surface. Therefore, the actual concentration would be less than the nominal one when the solution was at room temperature. Fortunately, these macro-objects had almost no effect on resultant SAMs as they would be spun away from the substrate with the medium in the spin-coating process. However, any microaggregates of OPA within the medium might result in aggregates on the resultant monolayer surface. This problem was seen for old solutions and could be remedied by using a freshly prepared solution or by heating the solution to $\sim 40{ }^{\circ} \mathrm{C}$. At these elevated temperatures, the OPA molecules appeared to be completely dissolved in the TCE solution. Temperature of the solution could be raised by placing the bottle containing the OPA solution in a warm water tank or on a hot plate. Other solvents were also used in search for the best available medium for achieving full-coverage OPA SAM.

Al films were coated on an n-type $\mathrm{Si}(100)$ substrate by $\mathrm{RF}$ magnetron-sputtering. The mica substrate was freshly cleaved, and other substrates including the n-type $\operatorname{Si}(100)$ substrate 
having a resistivity of $35-130 \mathrm{ohm}-\mathrm{cm}$ and the coated $\mathrm{Al}$ films were cleaned with methanol followed by being subjected to UV irradiation at primary lines at 184.9 and $253.7 \mathrm{~nm}$ with the presence of an ozone stream generated by a discharge ozone generator from a dry air flow $(\sim 1$ SLPM) for periods of $45-$ $60 \mathrm{~min}$. This UV/ozone (UVO) cleaning/oxidation served to remove organic contamination from the surface and to render it hydrophilic. ${ }^{27}$ Then, the freshly prepared OPA solution at room temperature was spin-coated on the substrate rotated at 3000-9000 rpm under a controlled relative humidity. Contamination on a substrate surface is thought to weaken or even block the interaction between the OPA headgroup and the substrate. Therefore, the treated substrate of interest should be used immediately for OPA SAM formation.

AFM. Dynamic force mode AFM (TopoMetrix's Explorer model) was used to image the OPA layers formed on a substrate. Rectangular-shaped silicon cantilevers with a spring constant of $\sim 40 \mathrm{~N} / \mathrm{m}$ were used in the dynamic force mode AFM. The cantilever was $125-\mu \mathrm{m}$ long, $35-\mu \mathrm{m}$ wide, and $4.0-\mu \mathrm{m}$ thick. The tip integrated on the free end of a cantilever had a nominal apex radius of $10 \mathrm{~nm}$. The dynamic force mode AFM measures the surface morphology through a feedback mechanism of maintaining constant damped oscillation amplitude while the vibrating tip scans the contour of the surface. The AFM scanner was calibrated using a height standard having a step height of $26 \pm 1 \mathrm{~nm}$. The images were obtained at a nominal scan speed of $5 \mu \mathrm{m} / \mathrm{s}$ under a relative humidity of $\sim 50 \%$. The data points in an AFM image were typically $400 \times 400$.

FTIR. Grazing-angle infrared (IR) reflection absorption spectra were obtained using a Fourier transform IR (FTIR) spectrometer (Bruker's IFS 55 model) equipped with a grazingangle reflectance accessory and a polarizer for the incident IR beam. The experiment was conducted on samples of OPA on a Si substrate and OPA on a commercial $\mathrm{Al}$ foil substrate having a lateral dimension of $1.5 \mathrm{~cm} \times 4.0 \mathrm{~cm}$ by measuring reflectance of the p-polarized IR beam with an $85^{\circ}$ angle of incidence. FTIR spectra were recorded at a $2 \mathrm{~cm}^{-1}$ resolution with 20000 averaged scans because of the weak absorbance detected using our instrument. The bare substrate was used as the background.

Contact Angle. A contact angle goniometer (Ramé-Hart's Model 100-00) was used to collect the data of contact angles for hexadecane and deionized water on the OPA samples having a full coverage. A sessile liquid drop of interest was used to measure the contact angles.

\section{Results and Discussion}

OPA/Mica. As mica is perhaps the most studied substrate for OPA SAM formation, we first describe our results on this system: Using a nonpolar medium, we have been able to readily prepare a full-coverage OPA SAM on cleaved mica substrates. Dynamic force mode AFM images in Figure $2 a-2 c$ show that the OPA SAM coverage increases up to $100 \%$ (Figure 2c) as aliquots of OPA are delivered to the mica surface. The apparent full coverage is judged from AFM images in an area of $1 \mu \mathrm{m}$ $\times 1 \mu \mathrm{m}$, which have a lateral spatial resolution of a couple of $\mathrm{nm}$. Therefore, any defects less than the AFM lateral resolution are not detectable by our AFM systems. Succeeding applications of the nonpolar solution to the OPA SAMs that have the morphology shown in Figure $2 \mathrm{~b}$ are able to fill the $\sim 30-\mathrm{nm}$ wide pits. For another type of mica, biotite, we also confirmed the ability to form full-coverage OPA SAM on it. On the basis of our extensive investigation on OPA SAM formation, we have found that the coverage is controllable by varying solution concentration, spin speed, and relative humidity. For example,

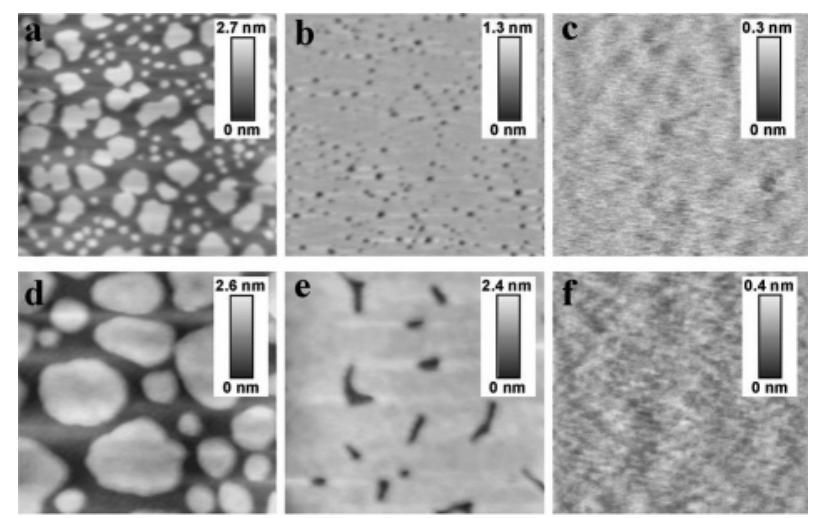

Figure 2. AFM images (scan area: $1 \mu \mathrm{m} \times 1 \mu \mathrm{m}$ ) for OPA SAMs delivered onto $(a-c)$ a freshly cleaved mica substrate and $(d-f)$ a 45 min UVO-treated Si substrate by applying increasing aliquots of a nominally $2 \mathrm{mM}$ trichloroethylene solution of OPA to the substrate rotated at $5000 \mathrm{rpm}$ under a relative humidity of $57 \%$ and $65 \%$ for the mica and the Si substrate, respectively. (c) and (f) show morphology of the SAM with $100 \%$ coverage. The height scale bar is inserted in the image.

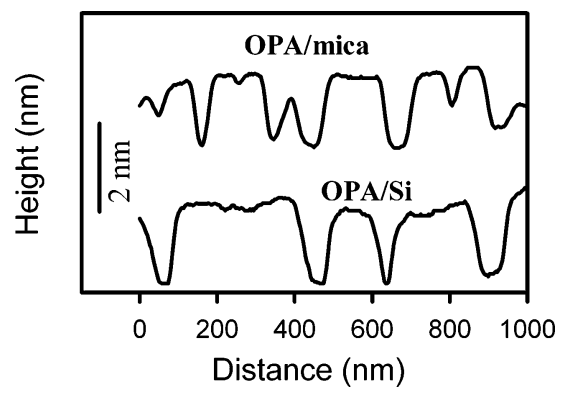

Figure 3. Profiles isolated from Figure $2 \mathrm{a}$ and $2 \mathrm{~d}$ for OPA SAMs formed on a mica and a $\mathrm{Si}$ substrate. The insert bar is $2 \mathrm{~nm}$.

we noticed that higher solution concentration and relative humidity rendered larger islands.

A profile from Figure $2 \mathrm{a}$ is shown in Figure 3. As seen in Figure 2a, there are occasional small "islands" that have a smaller thickness. Because the length of a fully extended OPA molecule is $2.5 \mathrm{~nm},{ }^{16}$ we conclude that the OPA molecules forming SAMs on the surface are tilted at a significant angle to it. The height of OPA SAMs on a mica substrate has been estimated to be $1.7 \pm 0.2 \mathrm{~nm}$ from analysis of numerous OPA/ mica samples.

OPA/Si. Shown in Figure 2d-2f are AFM images for OPA SAMs formed on an oxidized n-type $\mathrm{Si}(100)$ substrate. The $\mathrm{Si}$ substrate had been first cleaned in a UV/ozone system to render the surface hydrophilic by removing organic contamination from the surface. It was found that the SAM coverage could be increased to virtually $100 \%$ (Figure $2 \mathrm{f}$ ) by spin-coating successive aliquots of a nonpolar solution of OPA onto the surface. The increase in coverage on the Si substrate is shown in Figure $2 \mathrm{~d}-2 \mathrm{f}$ by imaging the surface between medium applications. Succeeding applications of the nonpolar medium continue to fill the unoccupied substrate with newly formed SAMs without disturbing the existing ones. We confirmed that OPA SAMs formed on a clean quartz substrate closely resembled those on the oxidized Si substrate.

The incomplete coverage shown in Figure 2a, 2b, 2d, and 2e is used to illustrate the additivity of the OPA SAM growth when a dilute OPA solution was used. When an adequately high concentration of OPA solution is used, only one application of the solution onto a rotated substrate renders a full-coverage OPA monolayer on the Si substrate. Dipping or immersing a UVO- 
treated Si substrate in an OPA solution in TCE was also able to produce OPA monolayers on the substrate. This shows that once the substrate is in contact with the OPA containing TCE medium, the OPA molecules concentrated on the medium surface will be transferred onto the hydrophilic substrate.

Shown in Figure 3 is a profile from Figure $2 \mathrm{~d}$ for displaying the OPA monolayer thickness. The thickness of the OPA SAMs on a $\mathrm{Si}$ substrate was estimated to be $1.8 \pm 0.2$ by averaging a large amount of thickness data we have obtained in our work of using AFM to characterize the SAM formation. This thickness estimation from the AFM results has been confirmed using spectroscopic ellipsometry, from which a full-coverage OPA SAM on a Si substrate has been estimated to have a thickness of $1.99 \pm 0.13 \mathrm{~nm}^{28}$

Other Solvents. When polar solvents, such as ethanol, were used, there was no formation of OPA SAMs at all on a $\mathrm{Si}$ substrate; instead, "liquidlike" multilayers were formed when the substrate was immersed in the solution, on which OPA molecules were observed to move around forming rodlike and crystalline features on a time scale of hours to days. ${ }^{18} \mathrm{We}$ also tried spin-coating OPA solution in ethanol, a polar solvent with a dielectric constant of $24.3,{ }^{29}$ on a Si substrate; however, instead of monolayer formation, observed were round features having a lateral dimension of 200-400 $\mathrm{nm}$ and a height of 4-6 nm, which are composed of smaller aggregates. This inability to form OPA SAMs from their solution in a hydrophilic medium ${ }^{18,25}$ might indicate that the weak interaction between the OPA headgroup and the Si substrate yields to other probably stronger interactions such as OPA molecular tail-tail interaction and head-head interaction.

Along with TCE, several other nonpolar solvents have been tested including chloroform, trichloroethane, dichloromethane, hexane, heptane, dodecane, and toluene; it appeared that only chloroform and TCE were able to deliver a full-coverage OPA SAM on an oxide. Therefore, it is clear that selection of solvent polarity is essential if one needs to have a full-coverage OPA monolayer free of pits. These are all nonpolar solvents but with different polarities, as indicated by their different dielectric constants. ${ }^{30,31}$ Trichloroethane and dichloromethane have dielectric constants of 7.5 and 9.1 , respectively; ${ }^{29}$ these tend to lead to the formation of aggregates, rather than monolayers on a Si substrate, as confirmed by AFM imaging. With a dielectric constant ranging from 1.9 to $2.4,{ }^{29}$ hexane, heptane, dodecane, and toluene did not appear to allow formation of good quality OPA SAMs on a Si substrate. The dielectric constants for TCE and chloroform, from which a full-coverage OPA SAM can be readily formed, are 3.4 and 4.8 , respectively. ${ }^{29}$ Therefore, a nonpolar solvent with a dielectric constant around 4 appears to deliver a full-coverage OPA monolayer on the mica surface. This suggests that a nonpolar solvent needs to have a suitable polarity to allow an adequate concentration of the OPA hydrophilic headgroup aligned at the medium surface, which is essential for OPA SAM formation on a Si substrate.

For solvents having a dielectric constant much higher than 4, they tend to dissolve the hydrophilic OPA headgroup within the medium instead of allowing the concentration of the headgroups on the medium surface. On the other hand, when a solvent has a dielectric constant substantially lower than 4 , they may tend to render the amphiphilic OPA molecules reversed micelles in the solution as well as to result in a deficiency in concentration of the hydrophilic OPA headgroups on the medium surface; both of these would not favor OPA SAM formation on a Si substrate. There are many solvent properties such as solubility and polarity that may have an impact on SAM
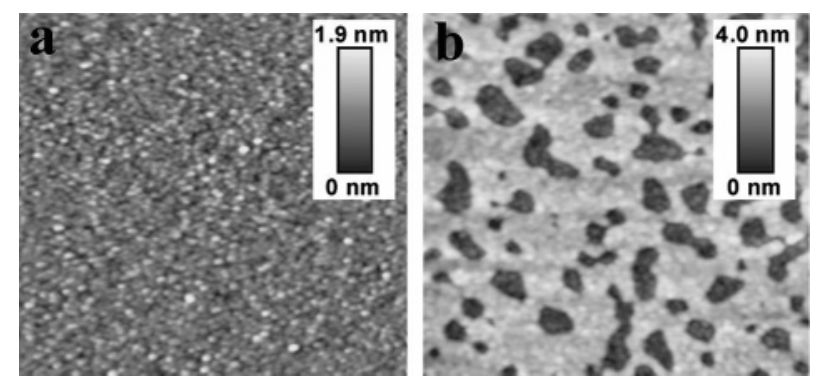

Figure 4. AFM images (scan area: $1 \mu \mathrm{m} \times 1 \mu \mathrm{m}$ ) show (a) the particle structure for a 10-nm-thick Al film sputter-coated on a Si substrate and (b) OPA SAMs delivered, under a relative humidity of $\sim 70 \%$, onto the $\mathrm{Al}$ film surface by allowing a $2 \mathrm{mM}$ trichloroethylene solution of OPA to stay on the UVO-treated Al film for $\sim 1$ min followed by spinning.

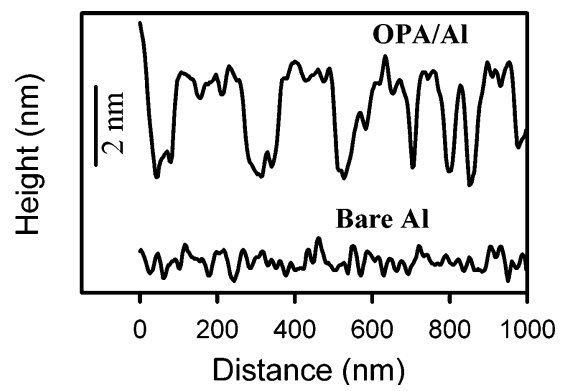

Figure 5. Typical profiles isolated from Figure 4 for the bare and OPA covered Al film. The insert bar is $2 \mathrm{~nm}$.

formation. ${ }^{10-12}$ We demonstrated here that the polarity as defined by dielectric constant plays a dominant role for the success of delivering OPA monolayers on a $\mathrm{Si}$ substrate.

OPA/Al. OPA SAMs have also been formed on an Al film that had been oxidized and cleaned using a UVO treatment for 50 min. Despite the roughness introduced by $\mathrm{Al}$ particles (Figure 4a) with an average corrugation height of $0.8 \mathrm{~nm}$, it appears that the OPA molecules in the SAM (Figure $4 \mathrm{~b}$ ) can follow the underlying corrugation where the corrugation height is shorter than the OPA molecular length. Shown in Figure 5 are two profiles each from Figure $4 \mathrm{a}$ and $4 \mathrm{~b}$ for the bare and OPA deposited Al films, respectively. The apparent thickness of the OPA layer is estimated to be $2.2 \pm 0.2 \mathrm{~nm}$ from the AFM image shown in Figure 4a; this suggests that OPA molecules are less tilted on Al than on mica and silicon.

For thicker Al films (e.g., 200 nm) deposited on a Si substrate as well as for Al plates and foils, the OPA monolayers were not able to be detected using AFM images because of rough surface features on these substrates. Although other methods, such as contact angle measurement, indicated that the treated surface was terminated by methyl groups, it was not possible to use AFM to visualize how OPA molecules are attached to these rough surfaces. Thus, there appears to be two critical conditions for the $\mathrm{Al}$ film that allow the AFM observation of OPA SAMs (Figure 4b): (1) UVO treatment and (2) surface corrugation less than the OPA molecular length. In Figure 4b, the lower coverage was chosen for display purposes; a full-coverage SAM on the oxidized $\mathrm{Al}$ film resembles the topography of the bare $\mathrm{Al}$ film itself. We believe that this is the first morphological evidence for formation of full-coverage OPA SAMs on a noncrystalline Al film; other analytical techniques have indicated the existence $\mathrm{e}^{20,22}$ and lubrication properties $^{21}$ of OPA monolayers deposited on oxidized Al films using the immersion method in either a polar or a nonpolar solvent.

It was also possible to form a full-coverage OPA SAM on a clean sapphire surface. As the sapphire substrate surface is 

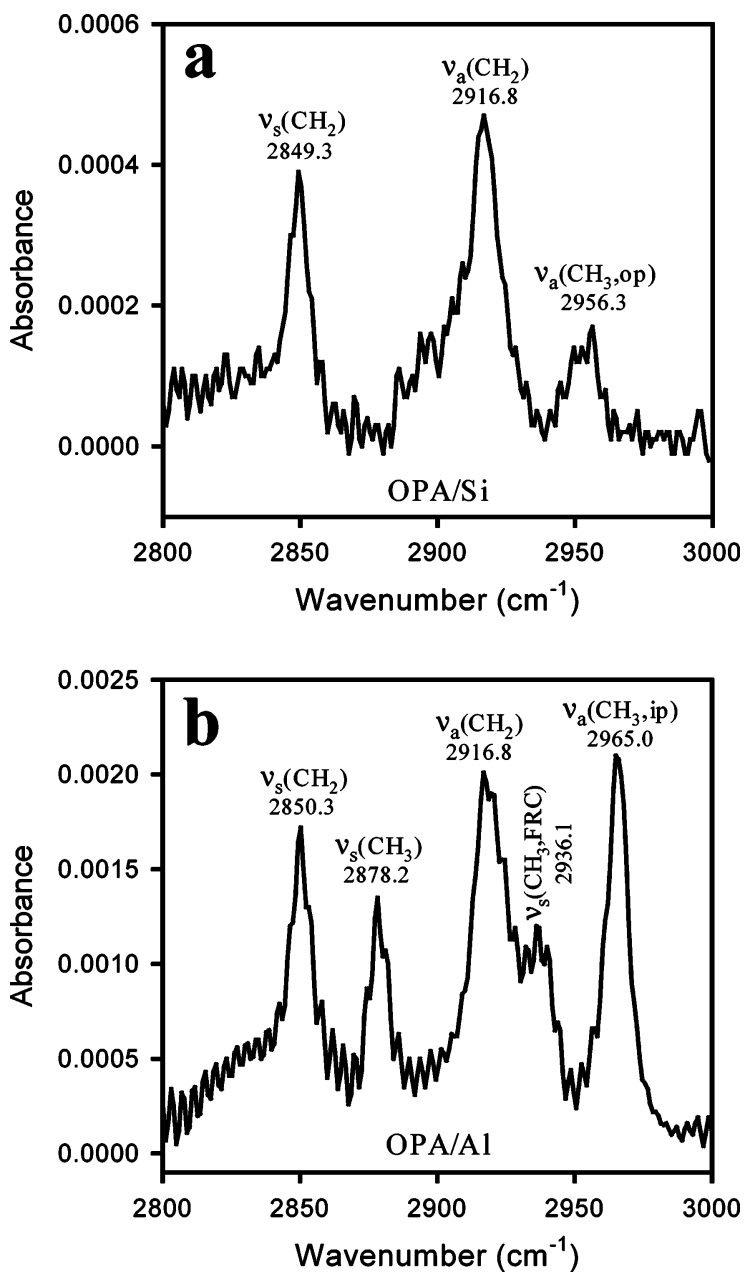

Figure 6. Grazing-angle FTIR absorption spectra for OPA SAMs spincoated, under a relative humidity of $\sim 70 \%$, on a 60 -min-UVO-treated (a) $\mathrm{Si}$ and (b) Al surface.

smooth, one can monitor the OPA coverage increase with increasing aliquots of the OPA solutions applied onto the substrate, in a similar way as those shown in Figure 2.

FTIR of OPA/Si and OPA/Al. The AFM images in Figures 2 and 4 show an apparently single-layered structure of the OPA molecules on three different oxide substrates. To verify the orderliness of the OPA SAMs produced with our method, we conducted FTIR absorption spectroscopy study on OPA samples prepared on a Si surface and an Al surface. For a molecular monolayer having backbone methylene chains, the peak frequencies in an IR spectrum for the symmetric and asymmetric methylene stretching modes, denoted as $v_{\mathrm{s}}\left(\mathrm{CH}_{2}\right)$ and $v_{\mathrm{a}}\left(\mathrm{CH}_{2}\right)$, respectively, have been established as a definitive means of distinguishing whether the methylene chains are an ordered (crystalline-like) structure or a disordered (liquidlike) structure. ${ }^{6,7,32-34}$ For a crystalline-like structure with all-trans methylene chain conformation, the peak frequencies for $v_{\mathrm{s}}\left(\mathrm{CH}_{2}\right)$ and $v_{\mathrm{a}}\left(\mathrm{CH}_{2}\right)$ appear at 2851 and $2918 \mathrm{~cm}^{-1}$, respectively, while a liquidlike structure of hydrocarbon chains is characterized with the two absorption bands shifting to higher frequencies, typically to 2855 and $2924-2929 \mathrm{~cm}^{-1}$, respectively. ${ }^{7,34} \mathrm{We}$ confirmed this by conducting a grazing-angle FTIR absorption measurement for a Vaseline (a typical example of a structure having highly disordered hydrocarbon chains) film spin-coated on a gold film, where $v_{\mathrm{s}}\left(\mathrm{CH}_{2}\right)$ and $v_{\mathrm{a}}\left(\mathrm{CH}_{2}\right)$ modes showed their peak frequencies at 2855.4 and $2927.1 \mathrm{~cm}^{-1}$, respectively.

The grazing-angle FTIR absorption spectrum for the OPA SAMs on a $\mathrm{Si}$ substrate is shown in Figure 6a. The peak frequencies of $v_{\mathrm{s}}\left(\mathrm{CH}_{2}\right)$ and $v_{\mathrm{a}}\left(\mathrm{CH}_{2}\right)$ for the OPA SAMs prepared on a $\mathrm{Si}$ substrate were found to be 2849.3 and $2916.8 \mathrm{~cm}^{-1}$, respectively. These two frequency positions for the symmetric and asymmetric methylene stretching modes indicate that the methylene chains in OPA SAMs on a Si substrate are closely

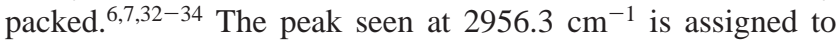
the out-of-plane asymmetric methyl stretching mode $\left[v_{\mathrm{a}}\left(\mathrm{CH}_{3}, \mathrm{op}\right)\right]$. The FTIR result for the OPA/Si shown in Figure 6a resembles those obtained for OTS SAMs on a $\mathrm{Si}^{2}$ or on a quartz ${ }^{3}$ substrate and chemically bonded alkyl monolayers ${ }^{35,36}$ on a hydrogenterminated $\mathrm{Si}(111)$ substrate. It is thus clear from the FTIR result shown in Figure 6a that on a Si substrate, OPA molecules form ordered monolayers having comparable quality to the wellinvestigated OTS SAMs on a silicon oxide. Also, the octadecyldihydrogen-phosphate $\left[\mathrm{CH}_{3}\left(\mathrm{CH}_{2}\right)_{17} \mathrm{OP}(\mathrm{O})(\mathrm{OH})_{2}\right]$ deposited on $\mathrm{ZnSe}$ or Ge surfaces ${ }^{2}$ has a similar IR absorption spectrum to that shown in Figure 6a.

It was difficult to perform the IR reflection absorption study on OPA SAMs on a mica substrate because of its low reflectivity, biaxial optical properties, and the occurrence of high-intensity interference fringes that overwhelm the signals from thin adsorbed films. ${ }^{37}$ However, some researchers were able to obtain transmission IR spectra for OTS SAMs on a mica substrate either under strict conditions of orientation of the mica substrate and the angle of incidence of the IR beam ${ }^{37}$ or by using an extremely thin ${ }^{38}$ (e.g., 1- $\mu$ m-thick) mica substrate. The IR spectra for OTS SAMs formed on a mica ${ }^{37,38}$ substrate resemble those on a $\mathrm{Si}^{2}$ substrate and the result shown in Figure 6a. We thus infer that the OPA monolayers on a mica substrate also have a similarly ordered structure as that prepared on a $\mathrm{Si}$ substrate.

We show in Figure $6 b$ the FTIR spectrum for OPA SAMs deposited on an Al foil surface, which had been cleaned using the same procedure as for the $\mathrm{Al} / \mathrm{Si}$ sample (the interface between a 10 -nm-thick $\mathrm{Al}$ film and the Si substrate seemed to cause spectral interference). The peaks at 2850.3 and 2916.8 $\mathrm{cm}^{-1}$ are assigned to $v_{\mathrm{s}}\left(\mathrm{CH}_{2}\right)$ and $\nu_{\mathrm{a}}\left(\mathrm{CH}_{2}\right)$, respectively. Once again, these peak frequencies are a signature for closely packed methylene chains. ${ }^{6,7,32-34}$ Also shown in the spectrum are the symmetric methyl stretching mode $\left[v_{\mathrm{s}}\left(\mathrm{CH}_{3}\right)\right]$ at $2878.2 \mathrm{~cm}^{-1}$ and its Fermi resonance splitting component $\left.\left[v_{\mathrm{s}}\left(\mathrm{CH}_{3}\right), \mathrm{FRC}\right)\right]$ at $2936.1 \mathrm{~cm}^{-1}$. The $v_{\mathrm{a}}\left(\mathrm{CH}_{3}, \mathrm{op}\right)$ is not determined as it may be buried within the shoulder of the strong $v_{\mathrm{a}}\left(\mathrm{CH}_{3}\right.$,ip) peak. The in-plane asymmetric methyl stretching mode $\left[v_{\mathrm{a}}\left(\mathrm{CH}_{3}, \mathrm{ip}\right)\right]$ with the peak frequency at $2965.0 \mathrm{~cm}^{-1}$ is the strongest peak in the spectrum. Alkanethiol SAMs formed on a silver surface have a much stronger $v_{\mathrm{a}}\left(\mathrm{CH}_{3}\right.$,ip) peak than that on a gold surface, which is attributed to a less tilt angle for the SAMs on the silver surface. ${ }^{39}$ Therefore, the IR absorption results shown in Figure 6 suggest that the OPA methylene chains are less tilted on an Al surface than on a Si substrate. The IR spectrum for the OPA/ Al sample shown in Figure $6 \mathrm{~b}$ resembles those obtained on $\mathrm{OTS}^{3}$ and $\mathrm{OPA}^{22}$ monolayers formed on an Al surface, where the $v_{\mathrm{a}}\left(\mathrm{CH}_{3}\right.$,ip) peak is the strongest.

Contact Angle Measurements. Knowing from the FTIR absorption spectra shown in Figure 6 that the methylene chains in the OPA SAMs are crystalline-like with all-trans conformation, ${ }^{7}$ we also verified the orderliness of the methyl groups terminating the monolayer by conducting contact angle measurements for OPA SAMs on mica, $\mathrm{Si}$, and $\mathrm{Al}$ surfaces using hexadecane (HD) because contact angles for HD on a surface is sensitive to the orderliness of methyl groups. ${ }^{5}$ Surfaces terminated with well-ordered methyl groups give an advancing contact angle of $\sim 45^{\circ}$ for HD. ${ }^{5}$ Our results are shown in Table 
TABLE 1: Contact Angles $\left(^{\circ}\right)$ for Hexadecane (HD) and Deionized Water

\begin{tabular}{rcccr}
\hline & $\begin{array}{c}\text { advancing } \\
\text { (HD) }\end{array}$ & $\begin{array}{c}\text { advancing } \\
\text { (water) }\end{array}$ & $\begin{array}{c}\text { receding } \\
\text { (water) }\end{array}$ & \multicolumn{1}{c}{$\begin{array}{c}\text { static } \\
\text { (water) }\end{array}$} \\
\hline OPA/mica & $46 \pm 1$ & $96 \pm 2$ & $<10$ & $60 \pm 5$ \\
OPA/Si & $46 \pm 1$ & $51 \pm 2$ & $<10$ & $38 \pm 3$ \\
OPA/Al/Si & $46 \pm 1$ & $111 \pm 1$ & $92 \pm 1$ & $101 \pm 1$
\end{tabular}

1 , in which the advancing HD contact angles are $\sim 46^{\circ}$ on a full-coverage OPA SAM prepared on all of the three substrates: the cleaved mica, UVO-treated $\mathrm{Si}$, and $\mathrm{Al} / \mathrm{Si}$ surfaces. Therefore, the OPA monolayers on the three substrates were terminated by well-ordered methyl groups. ${ }^{5,36} \mathrm{We}$ also confirmed using full-coverage OTS SAMs prepared on a $\mathrm{Si}$ substrate that the HD contact angle was also $46^{\circ}$ on the OTS SAM surface. This suggests that the orderliness of the methyl group in OPA SAMs on a Si substrate is comparable to that of the well-understood OTS SAMs.

Although the OPA SAMs display crystalline-like methylene chains and well-ordered methyl-terminating groups, as probed by FTIR and HD contact angle measurements, respectively, wetting properties probed by water were dramatically different among the SAMs formed on the three different substrates, as clearly shown in Table 1. For OPA SAMs on a mica surface, the advancing contact angle with water is $\sim 96^{\circ}$. The static water contact angle is $\sim 80^{\circ}$ at the beginning and reduces to $\sim 60^{\circ}$ within a few minutes. The receding contact angle with water is less than $10^{\circ}$, indicating that water has a strong interaction with the OPA SAMs. The wetting properties of the OPA SAMs prepared with the nonpolar solvent scheme were in good agreement with those prepared using the conventional polar solvent scheme, ${ }^{16}$ indicating that the two differently prepared SAMs have similar wetting properties. Although OPA SAMs prepared on a mica substrate appear to be mechanically robust as probed by scratch testing, ${ }^{19}$ they appear prone to water attack, suggesting that they are not chemically bonded to the substrate under the ambient conditions.

The advancing and static water contact angles for a fullcoverage OPA SAM on a Si substrate are even smaller than those observed on the OPA/mica sample: they are only $\sim 51^{\circ}$ and $\sim 38^{\circ}$, respectively. The receding water contact angle on the OPA SAMs is less than $10^{\circ}$. It is thus clear from the water contact angle measurement that water wets the surface of OPA SAMs formed on a $\mathrm{Si}$ substrate. Since the OPA monolayer is terminated by well-ordered methyl groups and has a closely packed methylene chain structure as determined by HD contact angle and FTIR spectrum, respectively, interactions between the OPA headgroup and the oxidized silicon surface must be weak to allow water attack. In fact, we observed partial removal of OPA molecules from their SAMs upon contact with water. Washing the surface with methanol for 5 min resulted in a removal of the vast majority of the OPA molecules from their SAMs on a Si substrate. These experimental facts thus suggest that the OPA headgroups are only physisorbed on the $\mathrm{Si}$ substrate. Since the OPA headgroup possesses a permanent dipole and the UVO-treated $\mathrm{Si}$ surface is terminated by hydroxide ions, we infer that the OPA molecules are most likely adsorbed onto the $\mathrm{Si}$ surface through an electric charge interaction or hydrogen bond. In addition, there may be sufficient free volume ${ }^{10}$ in the OPA SAMs to allow solvation of water, ${ }^{40}$ especially when one considers that phosphonic acid is very soluble in water. Although the OPA SAMs formed on a $\mathrm{Si}$ substrate are susceptible to alcohol and even water attack, they have an ordered structure from the methylene chains to the methyl termini as determined by the FTIR and the HD contact angle measurements, respectively, and their orderliness is comparable to other well-established SAM systems, such as OTS on a Si substrate. It appears, therefore, that our approach using an appropriate nonpolar solvent has provided a route to form a full-coverage OPA monolayer within seconds on an oxidized $\mathrm{Si}$ surface. This type of delicate OPA SAMs on a Si substrate may find applications in areas where easy removal of the monolayer template is required, for example, in lithography on the basis of the OPA/Si system.

On the other hand, for a full-coverage OPA SAM on a UVOtreated $\mathrm{Al}$ film, the advancing and receding water contact angles are $\sim 111^{\circ}$ and $\sim 92^{\circ}$, respectively. Water beads up on the OPA SAM surface, giving a static contact angle of $\sim 101^{\circ}$. The observed wetting behaviors suggest that the OPA SAMs on oxidized Al films have similar wetting properties to that of alkanethiol SAMs on a gold surface. ${ }^{5}$ We observed that the static contact angle did not change with time; the water bead maintained the same shape as it evaporated. Further, after the sample was sonicated in water and even in methanol for 10 min, no change was observed in the water contact angle. Therefore, it appears that OPA SAMs on oxidized Al films are not affected by water and alcohol attacks; thus, the interaction between the OPA headgroup and the oxidized Al film is likely of chemical bonding.

The drastic difference in wetting properties between the OPA/ $\mathrm{Al}$ and OPA/mica or OPA/Si systems suggests that the adsorption mechanism must be different. IR absorption studies showed that the methylene chains tilted less and AFM showed that the SAMs have a greater thickness on the Al surface than on the mica and Si surfaces. It is thus reasonable to assume that there is less free volume ${ }^{10}$ in the SAMs on the $\mathrm{Al}$ surface than on the mica or Si substrate. It has been known that phosphate or phosphonate groups make strong attachment to an oxidized $\mathrm{Al}$ film, most likely because of the formation of $\mathrm{P}-\mathrm{O}-\mathrm{Al}$ bonds. ${ }^{21,23,24}$

Fast Growth Rate of OPA SAMs. For OPA SAM formation on an oxidized Al surface, chemical bond formation ${ }^{23,24}$ between the $\mathrm{Al}$ and the oxygen atom from the phosphonate group releases a large amount of energy, accounting for the observed strong OPA SAMs. This appears consistent with the results from the calorimetric study by Ferreira et al., where they have reported a binding energy of $\sim 58 \mathrm{~kJ} / \mathrm{mol}$ for OPA monolayer formed on $\mathrm{TiO}_{2}{ }^{41}$ On the other hand, because OPA monolayers on $\mathrm{Si}$ substrates are prone to water attack and are washable by methanol, one infers that this is the result of the lack of chemical bond formation in the OPA/Si system. Such a delicate OPA monolayer on a Si substrate has thus to be formed via use of an appropriate nonpolar solvent that allows a direct transfer of aligned OPA headgroups at the nonpolar medium surface onto the Si substrate surface upon contact, which requires a very low activation energy to initiate the reaction for SAM formation. This environment also seems to be thermodynamically favorable for SAM formation as concentration of OPA headgroups on the medium surface is likely to reduce the entropy loss for SAM formation. ${ }^{42,43}$

We believe that our method provides a mechanism of lowering activation energy for SAM formation in that a "raft" of OPA molecules having the headgroup aligned on the nonpolar liquid medium surface is transferred onto the hydrophilic solid substrate upon physical contact, leading to an extremely fast monolayer formation. Growth rate is determined by the time the molecules take to contact the substrate; this is practically instantaneous. For an adequately high concentration, only one application of the solution is required to make a full-coverage 
OPA SAM on a substrate. On the other hand, for a lower concentration, more applications of the solution are necessary to make a full coverage. This nature of additivity of the OPA SAM growth in our method when using a lower concentration OPA solution can be used for coverage control.

We have noticed that an OPA containing TCE, upon contact with the UVO-treated Al film, appeared to be fiercely moving on the surface and the liquid medium often split to smaller droplets; this event appeared to last for $\sim 1 \mathrm{~s}$. Immediately after this event, the liquid medium stood still on the surface with a contact angle of $\sim 40^{\circ}$, indicating that the surface was now rendered hydrophobic, that is, the OPA SAMs had been formed. This phenomenon was also observed on the surface of cleaved mica and UVO-treated Si substrates.

We confirmed that a pure TCE solvent wetted these highsurface-energy substrates (a near-zero contact angle). Therefore, the observed short-lived violent behavior of the OPA containing TCE must bear information about the OPA SAM formation on a hydrophilic substrate. One has to realize that an OPA containing TCE medium is no longer a pure nonpolar solvent, rather, it becomes a medium loaded with oriented and concentrated OPA hydrophilic headgroups on its surface. The OPA containing TCE medium is supposed to wet the high-surfaceenergy substrate surface, that is, it has a near-zero contact angle with the substrate. However, OPA monolayers are being formed on the substrate immediately upon the contact between the medium and the substrate; these newly formed, low-surfaceenergy OPA monolayers (terminated by methyl groups) are now requiring the medium to have a contact angle of $\sim 40^{\circ}$. Consequently, the medium finds itself in a situation in which it tries to, on one hand, have a near-zero contact angle for the untouched (high-surface-energy) areas and, on the other hand, a $\sim 40^{\circ}$ contact angle for the once-touched (low-surface-energy areas because of formation of OPA SAMs) areas. The result of these two effects is the observed fierce movement of the OPA containing TCE medium on the hydrophilic substrate surface, trying to cover the hydrophilic substrate as widely as possible. This experimental observation demonstrates an extremely fast growth rate of OPA SAM formation when using an OPA solution in TCE.

Since Si wafer is the most important substrate in the electronic device industry, efforts have been made to form chemically bonded SAMs on the substrate. Hanson et al. have shown a method of carefully depositing OPA films on a Si substrate by immersing the substrate in a polar OPA solution and waiting for $3 \mathrm{~h}$ to allow the solvent to evaporate; then, the treated sample was heated at $140{ }^{\circ} \mathrm{C}$ for 2 days to strongly bond the OPA molecule as a phosphonate on the native oxide surface of the Si substrate. ${ }^{25}$ Their approach appears to promote a chemical reaction by annealing the OPA-covered $\mathrm{Si}$ substrate at a temperature well above OPA's melting point of $\sim 100{ }^{\circ} \mathrm{C}$. Linford et al. have also shown that alkyl monolayers could be chemically bonded on an oxygen-free, $\mathrm{H}$-terminated $\mathrm{Si}$ substrate when it is immersed in a molten organic acid. ${ }^{35,36}$ To obtain the final SAM, rinse is a necessary step to remove excess molecules from the surface. In comparison, our approach is to render a physisorbed OPA monolayer on a Si substrate at room temperature in a matter of seconds because of a lowered activation energy for OPA molecules to form SAMs on the Si substrate via use of an appropriate nonpolar solvent that forces hydrophilic OPA headgroups on its surface.

\section{Conclusions}

We demonstrated that selection of solvent polarity plays an important role in the formation of OPA SAM on oxide surfaces, as only the use of a nonpolar medium with a dielectric constant around 4, such as trichloroethylene and chloroform, renders a full coverage of the monolayer. In particular, we showed that solvent polarity determines whether OPA SAMs be formed up to $100 \%$ coverage (using nonpolar solvent trichloroethylene or chloroform) or not at all (using a polar solvent ethanol) on a $\mathrm{Si}$ substrate. The OPA SAMs that form on a Si wafer appear to be relatively weakly bonded to the substrate; by contrast, for oxidized Al surfaces a strong chemical bond is formed. We believe that the driving force for the formation of OPA SAMs having a weak interaction strength on a Si substrate is due to a decreased activation energy for SAM formation via use of an appropriate nonpolar solvent that allows hydrophilic OPA molecular headgroups to concentrate and align at the nonpolar liquid medium surface for rapid reaction with the substrate surface. This leads to a vastly improved control on OPA SAM formation and its coverage on a solid surface.

Acknowledgment. Part of this work was supported by a Western Innovation Fund from UWO. We thank B. Kobe and J. T. Francis for providing the magnetron-sputtered Al films on $\mathrm{Si}$.

Note Added after ASAP Publication. This Article was published on Articles ASAP on September 28, 2006. In the paragraph entitled "Fast Growth Rate of OPA SAMs", the word "increase" in the last sentence was changed to "loss". The corrected version was posted on September 29, 2006.

\section{References and Notes}

(1) Nuzzo, R. G.; Allara, D. L. J. Am. Chem. Soc. 1983, 105, 4481.

(2) Maoz, R.; Sagiv, J. J. Colloid Interface Sci. 1984, 100, 465. 201.

(3) Gun, J.; Iscovici, R.; Sagiv, J. J. Colloid Interface Sci. 1984, 101,

(4) Nuzzo, R. G.; Zegarski, B. R.; Dubois, L. H. J. Am. Chem. Soc. 1987, 109, 733

(5) Bain, C. D., Troughton, E. B.; Tao, Y. T.; Evall, J.; Whitesides, G. M.; Nuzzo, R. G. J. Am. Chem. Soc. 1989, 111, 321.

(6) Nuzzo, R. G.; Dubois, L. H.; Allara, D. L. J. Am. Chem. Soc. 1990 112, 558 .

(7) Porter, M. D.; Bright, T. B.; Allara, D. L.; Chidsey, C. E. D. J. Am. Chem. Soc. 1987, 109, 3559.

(8) Dubois, L. H.; Nuzzo, R. G. Annu. Rev. Phys. Chem. 1992, 43, 437.

(9) Whitesides, G. M.; Mathias, J. P.; Seto, C. T. Science 1991, 254, 1312 .

(10) Ulman, A. Chem. Rev. 1996, 96, 1533.

(11) Schwartz, D. K. Annu. Rev. Phys. Chem. 2001, 52, 107.

(12) Schreiber, F. Prog. Surf. Sci. 2000, 65, 151.

(13) Schreiber, F. J. Phys.: Condens. Matter 2004, 16, R881.

(14) Schwartz, D. K.; Steinberg, S.; Israelachvili, J.; Zasadzinski, J. A. N. Phys. Rev. Lett. 1992, 69, 3354 .

(15) Bierbaum, K.; Grunze, M.; Baski, A. A.; Chi, L. F.; Schrepp, W.; Fuchs, H. Langmuir 1995, 11, 2143.

(16) Woodward, J. T.; Ulman, A.; Schwartz, D. K. Langmuir 1996, 12, 3626.

(17) Neves, B. R. A.; Salmon, M. E.; Russell, P. E.; Troughton, E. B., Jr. Langmuir 2000, 16, 2409 .

(18) Nie, H.-Y.; Walzak, M. J.; McIntyre, N. S. Langmuir 2002, 18 2955

(19) Nie, H.-Y.; Miller, D. J.; Francis, J. T.; Walzak, M. J.; McIntyre, N. S. Langmuir 2005, 21, 2773.

(20) Gao, W.; Dickinson, L.; Grozinger, C.; Morin, F. G.; Reven, L. Langmuir 1996, 12, 6429.

(21) Berman, A.; Steinberg, S.; Campbell, S.; Ulman, A.; Israelachvili, J. Tribol. Lett. 1998, 4, 43.

(22) Liakos, I. L.; Newman, R. C.; McAlpine, E.; Alexander, M. R. Surf. Interface Anal. 2004, 36, 347.

(23) Pellerite, M. J.; Dunbar, T. D.; Boardman, L. D.; Wood, E. J. J. Phys. Chem. B 2003, 107, 11726.

(24) Ramsier, R. D.; Henriksen, P. N.; Gent, A. N. Surf. Sci. 1988, 203,

(25) Hanson, E. L.; Schwartz, J.; Nickel, B.; Koch, N.; Danisman, M. F. J. Am. Chem. Soc. 2003, 125, 16074.

(26) Messerschmidt, C.; Schwartz, D. K. Langmuir 2001, 17, 462. 
(27) Zazzera, L.; Evans, J. F. J. Vac. Sci. Technol., A 1993, 11, 935.

(28) Private communication from Dr. Jieming Feng of Nanometrics Inc., Tokyo, Japan in July 2005.

(29) Gokel, G. W. Dean's Handbook of Organic Chemistry, 2nd ed; McGraw-Hill: New York, 2004.

(30) Gratzer, P. F.; Pereira, C. A.; Lee J. M. J. Biomed. Mater. Res. 1996, 31, 533

(31) Drain, C. M.; Gentemann, S.; Roberts, J. A.; Nelson, N. Y.; Medforth, C. J.; Jia, S. L.; Simpson, M. C.; Smith, K. M.; Fajer, J.; Shelnutt, J. A.; Holten, D. J. Am. Chem. Soc. 1998, 120, 3781.

(32) Allara, D. L.; Swalen, J. D. J. Phys. Chem. 1982, 86, 2700.

(33) Snyder, R. G.; Strauss, H. L.; Elliger, C. A. J. Phys. Chem. 1982 $86,5145$.

(34) Tillman, N.; Ulman, A.; Schildkraut, J. S.; Penner, T. L. J. Am. Chem. Soc. 1988, 110, 6136.

(35) Linford, R. M.; Chidsey, C. E. D. J. Am. Chem. Soc. 1993, 115 12631
(36) Linford, R. M.; Fenter, P.; Eisenberger, P. M.; Chidsey, C. E. D. J. Am. Chem. Soc. 1995, 117, 3145 .

(37) Carson, G.; Granick, S. Appl. Spectrosc. 1989, 43, 473-476.

(38) Guzonas, D. A.; Hair, M. L.; Tripp, C. P. Appl. Spectrosc. 1990, 44, 290 .

(39) Laibinis, P. E.; Whitesides, G. M.; Allara, D. L.; Tao, Y. T.; Parikh, A. N.; Nuzzo, R. G. J. Am. Chem. Soc. 1991, 113, 7152.

(40) Mrksich, M.; Whitesides, G. M. Annu. Rev. Biophys. Biomol. Struct. 1996, 25, 55.

(41) Ferreira, M. J.; Marcinko, S.; Sheardy R.; Fadeev, A. Y. J. Colloid Interface Sci. 2005, 286, 258.

(42) Searle, M. S.; Williams, D. H. J. Am. Chem. Soc. 1992, 114, 10690-10697.

(43) Schessler, H. M.; Karpovich, D. S.; Blanchard, G. J. J. Am. Chem Soc. 1996, 118, 9645-9651. 